tion. Moreover, since he allows $m$ to take any value less than $P$, there may be several fractional values of $m$ corresponding to a single integer value of $F$; thus in his Tables I and IV the single pattern which I described $[4,5]$ as $15 / 3 / 1$ is variously described as $(15,3,1 / 5),(15,3$, $4 / 5),(15,3,7 / 5)$ and $(15,3,13 / 5)$, while the complementary retrograde orbit pattern $15 / 3 / 2$ is described as $(15,3,2 / 5),(15,3,8 / 5),(15,3,11 / 5)$ and $(15,3,14 / 5)$.

4) Ballard says that "Walker indicates that at least 10 satellites are required for triple visibility and 13 for quadruple visibility." I was actually presenting results only for patterns which would provide a positive minimum elevation angle with satellites in $24 \mathrm{~h}$ orbits, for from single to sevenfold coverage, and so those were the smallest numbers for which I presented $[4,5]$ triple and quadruple coverage results. Ballard's Theorem I provides a useful guide; however, as he himself comments, the patterns 9/9/7 and $11 / 11 / 4$ listed in his Table III as providing triple and quadruple visibility, respectively, might not be very practical, since they would not provide positive minimum elevation angles at altitudes less than about double the synchronous altitude.

5) Though Ballard apparently disagrees, at least as regards single coverage, with my view that the minimum satellite separation is an important characteristic of a pattern second only to the degree of coverage provided, I would expect that the zero minimum satellite separation associated with the pattern 10/10/7 quoted by Mozhayev [7] and Ballard would disqualify it from adoption for any practical system and that pattern $10 / 5 / 2$, having $[4,5]$ the slightly inferior maximum single-coverage radius of $52.2^{\circ}$ but a minimum satellite separation of $46.6^{\circ}$, would be preferred. Incidentally, Ballard's Fig. 11(B) provides a particularly clear illustration of the concept of minimum satellite separation, with no other satellite passing near satellite 0 at any time.

6) I feel that one of the more useful results from my studies, in addition to those mentioned by Ballard, was the development of a simple method of identifying which pattern would be found, for a particular number of satellites, to provide the largest minimum satellite separation; this would often prove also to be the pattern which provided the smallest maximum $n$-coverage radius, but even when this was not the case it would provide a radius close to the smallest. Briefly, this method $[5,6]$ is as follows. For a total of $T$ satellites, the selected pattern is $T / P / F$, where $P$ (the number of orbital planes) is equal to $T$ divided by the highest common factor of $T$ and $M ; F$ (a measure of the relative phasing between planes, in units of $\left.360^{\circ} / T\right)$ is equal to $T(k P-L) / P M ; L: M$ equals $2: 1$ for values of $T$ from 5 to $9,3: 2$ for $T$ from 10 to 24 , and $4: 3$ from 25 upwards; and $k$ takes whatever integer value is necessary to make $F$ an integer in the range 0 to $P-1$. Thus, for $T=9$ and 11 , respectively, this method identifies the patterns $9 / 9 / 7$ and $11 / 11 / 4$ mentioned in comment
(4), while for $T=10$ it identifies the pattern $10 / 5 / 2$ mentioned in comment (5). Other useful patterns can be found by using the same values of $L: M$ over a wider range of values of $T$; for example, patterns $5 / 5 / 1$ and $7 / 7 / 2$, listed in Ballard's Table III, are found by using $L: M=3: 2$, and pattern $18 / 6 / 2$, mentioned [5] as of potential interest for systems such as GPS, is found by using $L: M=4: 3$.

\section{J.G. WALKER}

Royal Aircraft Establishment Space Department Farnborough Hants GU14 6TD England

\section{REFERENCES}

[1] Ballard, A. H. (1980) Rosette constellations of earth satellites IEEE Transactions on Aerospace and Electronic Sistems, Sept. 1980, 16, 656-673.

[2] Walker. J. G. (1970) Circular orbit patterns providing continuous whole Earth coverage.

Technical Report 70211, Royal Aircraft Establishment, Farnborough, England.

[3] Walker, J. G. (1971)

Some circular orbit patterns providing continuous whole earth coverage.

Journal of the British Interplanetary Society. July 1971, 24. 369-384.

[4] Walker. J. G. (1973)

Continuous whole-earth coverage by circular-orbit satellites. IEE Conference Publication 95. "Satellite systems for mobile communications and surveillance," 1973. pp. 35-38.

[5] Walker, J. G. (1977)

Continuous whole-earth coverage by circular-orbit satellite patterns.

Technical Report 77044. Royal Aircraft Establishment. Farnborough, England.

[6] Walker, J. G. (1978)

Satellite patterns for continuous multiple whole-earth coverage.

IEE Conference Publication 160, “'Maritime and aeronautical satellite communication and navigation," 1978, pp. 119-122

Mozhayev, G. V. (1973)

The problem of continuous earth coverage and kinematically regular satellite networks, pt. II.

Kosmicheskie Issledovaniya. Jan.-Feb. 1973, I1, 59-69

\section{Multichannel Recovery of Quadrature Components of Bandpass Signals}

The problem of sampling signals maintaining the theoretically minimum (average) sampling rate and allowing a separate interpolation is considered from a general point of view. The formulation will follow a recently published method for multichannel sampling; this way there is the advantage of working with functions depending only on the frequency and not on time and frequency as in other approaches. This simplifies the general expressions and the determi-

Manuscript received October 16, 1981. 
nation of separation conditions. Under the assumption of nonsingularity of certain matrices, we obtain the necessary and sufficient conditions for a separate interpolation and discuss them. Finally, an especially important case is considered as an example.

\section{INTRODUCTION}

Bandpass signal sampling is a subject in which some aspects are still under investigation. A direct sampling requires a rate between $\sigma / \pi$ and $2 \sigma / \pi$ (where $\sigma$ is the angular bandwidth of the signal) to allow a posterior interpolation of the original signal [1]. Two classical approaches to maintain the (average) sampling rate at its (theoretical) minimum value are

1) to sample the in-phase and quadrature components of the signal $i_{x}$ and $q_{x}$ in

$x(t)=i_{x}(t) \cos \omega_{0} t-q_{x}(t) \sin \omega_{0} t$

(where $\omega_{0}$ is the center angular frequency) that are lowpass, $\sigma / 2$-band-limited signals, according to the usual sampling theorem $[2,3]$; therefore, at a rate $\sigma /$ $2 \pi$ each;

2) to sample (at the same rate $\sigma / 2 \pi$ ) $x$ and its Hilbert transform

$\hat{x}(t) \triangleq 1 / \pi t * x(t)=\int_{-x}^{\infty} x\left(t-t^{\prime}\right) d t^{\prime} / \pi t^{\prime}$

where $*$ indicates convolution [4].

These two methods show similar drawbacks: the obtaining of $i_{x}, q_{x}$, or $\hat{x}$. Additionally, the second of them does not offer in a general case a separate interpolation, i.e., the possibility of obtaining $i_{x}\left(q_{x}\right)$ from an interpolation of only the set of samples from one signal. This limitation is common with the general version of another well-known bandpass sampling method, that of Kohlenberg $[5,6]$, which can be viewed as a sampling of $x(t)$ and $x\left(t-t_{0}\right), t_{0} \neq \pi / \sigma$.

Grace and Pitt [7] have introduced a separate interpolation formula based on sampling $x(t)$ and $x\left(t-\pi / 2 \omega_{0}\right)$ at $\sigma / 2 \pi$ each, valid, as Persons computes [8] and Brown proves [9-11], when $\omega_{0}=k \sigma / 2, k$ an integer. The same condition implies a separate interpolation when $x, \hat{x}$ are sampled.

In a first step of our work on bandpass sampling, we have shown that a separate interpolation is obtainable from samples of the outputs of two linear, time-invariant systems driven by $x$, when $\omega_{0}=k \sigma / 2$, if and only if the impulse response of one system has a zero quadrature component and the other has a zero in-phase component ( $x$ and $\hat{x}$ case excepted). This possibility has been proved following two approaches: the first [12], following Linden's [6] proof of Kohlenberg's second-order sampling [5]; the second [13], modifying Papoulis' generalized sampling expansion [14-16] to cover separate bandpass sampling. The same result is obtained, but the two proofs offer different ways to compute the interpolating functions for $i_{x}$ and $q_{x}$
In [12] and [13] we have indicated that $\omega_{0} \neq k \sigma / 2$ implies, for a general $x$, bandpass interpolating functions with spectral discontinuities, and this fact prevents a separate interpolation. The alternative to sampling $x$ and $\hat{x}$ necessitates the same condition; but its analysis does not follow the same formulation.

In a second step [17], and considering that the reasoning of the previous paragraph can be immediately extended, we have generalized our modification of Papoulis' generalized sampling expansion to sample at a rate $\sigma / 2 \pi N$ the outputs of $2 N$ linear time-invariant systems driven by $x$, obtaining a general separate interpolation formula when $\omega_{0}=k \sigma / 2 N$ (generalized Brown's condition) and $N$ systems have zero quadrature component impulse responses and the other $N$ have zero inphase component impulse responses. Interpolating functions are obtained from the functions $\left\{Y_{i}(\omega, t)\right\}, i=1$, $\ldots, 2 N$, solutions of the system

$$
\begin{aligned}
& \sum_{i=1}^{2 N} H_{i}(\omega+r c) Y_{i}(\omega, t)=\exp (j r c t), \\
& r=0, \ldots, N-1 \\
& \sum_{i=1}^{2 N} H_{i}\left(\omega+2 \omega_{0}+r c\right) Y_{i}(\omega, t)=\exp \left[j \left(2 \omega_{0}\right.\right. \\
& +r c) t], \quad r=0, \ldots, N-1
\end{aligned}
$$

(equivalent to $\left[16\right.$, eq. (7)]), where $c=\sigma / N$ and $\left\{H_{i}(\omega)\right\}$, $i=1, \ldots, 2 N$, are the transfer functions of the $2 N$ systems. We require that $(3)$ offers unique solutions $\left\{Y_{i}(\omega\right.$, $t)\}, i=1, \ldots, 2 N$. Cases in which $N$ systems and the Hilbert transforms of their inputs are used, which do not provide unique solutions for (3), as one checks easily by introducing an equivalent low pass formulation (see [17]), can be considered by an immediate generalization of the procedure introduced in [10].

Formula (3) can be considered as a useful particular case of a generalized bandpass signal sampling introduced by Brown [18].

Recently Brown $[19,20]$ has derived Papoulis' generalized sampling expansion formulas and their extension for bandpass sampling according to different reasoning, obtaining directly the Fourier transforms of the interpolating functions, i.e., the transfer functions of the interpolating filters, using a pieced calculation. In this paper this simpler and advantageous solution is used to introduce the separate interpolation, and a generalized Brown's condition is discussed. A particularly important case is shown as an example.

\section{RESTRICTED VERSION OF MULTICHANNEL BANDPASS SAMPLING}

We introduce a restricted version of Brown's multichannel bandpass sampling theorem which is useful in obtaining a separate interpolation formula. We omit the

0018-9251/82/1100-0725\$00.75 (C) 1982. 
corresponding proof, an immediate particularization of that contained in [20].

Let $x$ be a deterministic, real, finite-energy, bandpass signal, i.e.,

$$
\begin{aligned}
\int_{-\infty}^{\infty} x^{2}(t) d t & =\frac{1}{2 \pi} \int_{\omega_{0}-\sigma / 2<|\omega|<\omega_{0}+\sigma / 2}|X(\omega)|^{2} d \omega \\
& =E(x)<x \\
X(\omega) & =X^{*}(-\omega)
\end{aligned}
$$

where $X$ is the Fourier transform of the signal, $E$ is its energy, $\omega_{0}$ is its center angular frequency, and $\sigma$ is its angular bandwidth. Let $\left\{H_{i}(\omega)\right\}, i=1, \ldots, 2 N$, be the transfer functions of $2 N$ real, linear, time-invariant systems. It is possible to write

$x(t)=\sum_{i=1}^{2 N} \sum_{n=-\infty}^{\infty} g_{i}(n T) y_{i}(t-n T)$

where $T=2 \pi N / \sigma$ and $g_{i}$ is the output of the $i$ th system (driven by $x$ ). Assuming that $\omega_{0}=k \sigma / 2 N$, the Fourier transforms $\left\{Y_{i}(\omega)\right\}, i=1, \ldots, 2 N$, of the interpolating functions $\left\{y_{i}(t)\right\}, i=1, \ldots, 2 N$. can be obtained for $\omega>$ 0 (they are Hermitian functions) from the $N$ svstems

$$
\begin{gathered}
\sum_{i=1}^{2 N} Y_{i}(\omega+l c) H_{i+}(\omega+p c) \equiv 0, \\
p=0, \ldots, l-1 \\
\sum_{i=1}^{2 N} Y_{i}(\omega+l c) H_{i+}(\omega+l c) \equiv T \\
\sum_{i=1}^{2 N} Y_{i}(\omega+l c) H_{i+}(\omega+p c) \equiv 0, \\
p=l+1, \ldots, N-1 \\
\sum_{i=1}^{2 N} Y_{i}(\omega+l c) H_{i} \ldots[\omega-(k-p) c] \equiv 0, \\
p=0, \ldots, N-1
\end{gathered}
$$

$l=0, \ldots, N-1$, where $\omega_{0}-\sigma / 2<\omega<\omega_{0}-\sigma / 2+c, c$ $=\sigma / N=2 \pi / T, H_{i+}(\omega)=H_{i}(\omega) u(\omega), H_{i-\ldots}(\omega)=$ $H_{i}(\omega) u(-\omega)$, and $u$ is the unit step function. For each $l$ we have $2 N$ equations with $2 N$ unknowns, $\left\{Y_{i}(\omega+l c)\right\}, i$ $=1, \ldots, 2 N$; assuming that systems (7) offer unique solution sets, these determine the spectra of the $2 \mathrm{~N}$ interpolating functions (i.e., the transfer functions of the interpolating filters). It is possible to present (7) completely in a matrix formulation; but we prefer to establish the equivalent low pass equations before using this kind of formulation.

\section{EQUIVALENT LOW PASS FORMULATION}

The complex envelopes of $\left\{y_{i}(t)\right\}, i=1, \ldots, 2 N$, and of $\left\{h_{i}(t)\right\}, i=1, \ldots, 2 N\left(h_{i}\right.$ is the impulse response of

$0018-9251 / 82 / 1100-0726 \$ 00.75$ C 1982 the $i$ th system, i.e., the inverse Fourier transform of $H_{i}$ ) have spectra [21]

$B_{y i}(\omega)=2 Y_{i}\left(\omega+\omega_{0}\right) u\left(\omega+\omega_{0}\right)$
$B_{h_{i}}(\omega)=2 H_{i}\left(\omega+\omega_{0}\right) u\left(\omega+\omega_{0}\right)$

respectively; using these functions and keeping in mind that

$H_{i}(\omega)=H_{i}^{*}(-\omega)$

systems (7) become

$\sum_{i=1}^{2 N} B_{y_{i}}(\omega+l c) B_{h_{i}}(\omega+p c) \equiv 0$,

$p=0, \ldots, l-1$

$\sum_{i=1}^{2 N} B_{y_{i}}(\omega+l c) B_{h_{i}}(\omega+l c) \equiv 4 T$

$\sum_{i=1}^{2 N} B_{y_{i}}(\omega+l c) B_{h_{i}}(\omega+p c) \equiv 0$,

$$
p=l+1, \ldots, N-1
$$

$\sum_{i=1}^{2 N} B_{y_{i}}(\omega+l c) B_{h_{i}}^{*}[-(\omega+p c)] \equiv 0$,

$$
p=0, \ldots, N-1
$$

$l=0, \ldots, N-1$, in $-\sigma / 2<\omega<-\sigma / 2+c .\left\{B_{y_{i}}(\omega)\right\}$, $i=1, \ldots, 2 N$, are obtained from these pieced equations. Their Hermitian and anti-Hermitian parts are the Fourier transforms of the in-phase and quadrature components, respectively, of the $\left\{y_{i}(t)\right\}, i=1, \ldots, 2 N$.

\section{MATRIX FORMULATION}

All the equations implied by (10) can be written in a matrix form, introducing a $2 N \times N$ matrix $\boldsymbol{B}$ and a $2 N$ $\times 2 N$ matrix $\boldsymbol{A}$ having elements

$$
\begin{aligned}
\boldsymbol{B}]_{m . n}= & B_{y_{m}}[\omega+(n-1) c], \\
& 1 \leqslant m \leqslant 2 N, 1 \leqslant n \leqslant N
\end{aligned}
$$

and

$$
\boldsymbol{A}]_{m, n}=\left\{\begin{array}{l}
\boldsymbol{B}_{h_{n}}[\omega+(m-1) c] \\
1 \leqslant m \leqslant N, \leqslant n \leqslant 2 N \\
\boldsymbol{B}_{h_{n}}^{*}[-\omega-(m-N-1) c] \\
N+1 \leqslant m \leqslant 2 N, 1 \leqslant n \leqslant 2 N
\end{array}\right.
$$

respectively, and the $2 N \times N$ matrix

$$
\boldsymbol{T}=\left[\begin{array}{cc}
4 T & \boldsymbol{I}_{N} \\
\boldsymbol{O}_{N} &
\end{array}\right]
$$


where $\boldsymbol{I}_{N}$ is the $N \times N$ identity matrix, and $\mathbf{0}_{N}$ is the $N$ $\times N$ null matrix. Then (10) becomes

$A B=T$

in $-\sigma / 2<\omega<-\sigma / 2+c$, and, if $\boldsymbol{A}$ is nonsingular, we have

$\boldsymbol{B}=\boldsymbol{A}^{-1} \boldsymbol{T}$

in $-\sigma / 2<\omega<-\sigma / 2+c$. Each row of $\boldsymbol{B}$ defines an interpolating function (offers the spectra of its in-phase and quadrature components).

\section{TO OBTAIN A SEPARATE INTERPOLATION}

Since

$$
\begin{aligned}
y_{i}(t-n T)= & i_{y_{i}}(t-n T) \cos \left[\omega_{0}(t-n T)\right] \\
& -q_{Y_{i}}(t-n T) \sin \left[\omega_{0}(t-n T)\right] \\
= & (-1)^{n k} i_{y_{i}}(t-n T) \cos \omega_{0} t \\
& -(-1)^{n k} q_{y_{i}}(t-n T) \sin \omega_{0} t
\end{aligned}
$$

we need $q_{y_{i}} \equiv 0$ or $i_{y_{i}} \equiv 0$ to obtain separate contributions to the final interpolation formula; equivalently, $B_{y_{i}}(\omega) \equiv B_{y_{i}}^{*}(-\omega)$ or $B_{y_{i}}(\omega) \equiv-B_{y_{i}}^{*}(-\omega)$. It is clear from (14) that this will be possible if and only if there are $N$ Hermitian and $N$ anti-Hermitian $B_{h_{i}}$; since indices are unimportant, we assume

$B_{h_{i}}(\omega)=B_{h_{i}}^{*}(-\omega), \quad i=1, \ldots, N$

and

$B_{h_{i}}(\omega)=B_{h_{i}}^{*}(-\omega), \quad i=N+1, \ldots, 2 N$.

These conditions are equivalent to $q_{h_{i}}=0, i=1$, $\ldots, N$, and $i_{h_{i}}=0, i=N+1, \ldots, 2 N$; or to even/odd symmetry of $\operatorname{Re}\left[H_{i}(\omega) u(\omega)\right] / \operatorname{Im}\left[H_{i}(\omega) u(\omega)\right](i=1$, $\ldots, N)$ or of $\operatorname{Im}\left[H_{i}(\omega) u(\omega)\right] / \operatorname{Re}\left[H_{i}(\omega) u(\omega)\right](i=N+$ $1, \ldots, 2 N)$ with respect to $\omega_{0}$, respectively.

Under these assumptions we can add the $n$th and $(N$ $+n)$ th equations of (14), or subtract the $(N+n)$ th from the $n$th equation $(n=1, \ldots, N)$, obtaining the equivalent systems

$\boldsymbol{A}_{r 1} \boldsymbol{B}_{r 1}=2 T \boldsymbol{I}_{N}$

$\boldsymbol{A}_{r 2} \boldsymbol{B}_{r 2}=2 T \boldsymbol{I}_{N}$

in $-\sigma / 2<\omega<-\sigma / 2+c$, where matrices $\boldsymbol{A}_{r 1}, \boldsymbol{A}_{r 2}$, $\boldsymbol{B}_{r 1}, \boldsymbol{B}_{r 2}$, have elements

$$
\begin{aligned}
\left.\boldsymbol{A}_{r 1}\right]_{m, n}= & B_{h_{n}}[\omega+(m-1) c], \\
& 1 \leqslant m, n \leqslant N \\
\left.\boldsymbol{A}_{r 2}\right]_{m, n}= & B_{h_{n+N}}[\omega+(m-1) c], \\
& 1 \leqslant m, n \leqslant N \\
\left.\boldsymbol{B}_{r 1}\right]_{m, n}= & B_{y_{m}}[\omega+(n-1) c], \\
& 1 \leqslant m, n \leqslant N
\end{aligned}
$$

$$
\begin{gathered}
\left.\boldsymbol{B}_{r 2}\right]_{m, n}=B_{y_{m+N}}[\omega+(n-1) c], \\
1 \leqslant m, n \leqslant N .
\end{gathered}
$$

Naturally the $\left\{B_{y_{i}}(\omega)\right\}, i=1, \ldots, 2 N$, are obtained from

$\boldsymbol{B}_{r 1}=2 T \boldsymbol{A}_{r 1}^{-1}$

$\boldsymbol{B}_{r 2}=2 T \boldsymbol{A}_{r 2}^{-1}$

in $-\sigma / 2<\omega<-\sigma / 2+c$, assuming that $A_{r 1}, A_{r 2}$ are nonsingular. The final separate interpolation formula is

$$
\begin{aligned}
x(t)= & \left\{\sum_{i=1}^{N} \sum_{n=-\infty}^{\infty}(-1)^{k n} g_{i}(n T) i_{y_{i}}(t-n T)\right\} \\
& \cdot \cos \omega_{0} t-\left\{\sum_{i=N+1}^{2 N} \sum_{n=-\infty}^{\infty}(-1)^{k n} g_{i}\right. \\
& \left.\cdot(n T) q_{y_{i}}(t-n T)\right\} \sin \omega_{0} t .
\end{aligned}
$$

\section{AN ILLUSTRATIVE EXAMPLE}

Let us select $N=2$; then

$$
\begin{aligned}
& g_{1}(t)=x(t) \\
& g_{2}(t)=x\left(t-\pi / \omega_{0}\right) \\
& g_{3}(t)=x\left(t-\pi / 2 \omega_{0}\right) \\
& g_{4}(t)=x\left(t-3 \pi / 2 \omega_{0}\right) .
\end{aligned}
$$

We have $c=\sigma / 2, T=2 \pi N / \sigma=\pi N / c=2 \pi / c$, and $\omega_{0}=k \sigma / 4=k c / 2$. The spectra of the complex envelopes of the corresponding impulse responses are

$B_{h_{1}}(\omega)=2$

$B_{h_{2}}(\omega)=2 \exp \left[-j\left(\omega+\omega_{0}\right) \pi / \omega_{0}\right]=$

$$
-2 \exp \left(-j \omega \pi / \omega_{0}\right)
$$

$$
B_{h_{3}}(\omega)=2 \exp \left[-j\left(\omega+\omega_{0}\right) \pi / 2 \omega_{0}\right]=
$$

$$
-2 j \exp \left(-j \omega \pi / 2 \omega_{0}\right)
$$

$$
\begin{aligned}
B_{h_{4}}(\omega)= & 2 \exp \left[-j\left(\omega+\omega_{0}\right) 3 \pi / 2 \omega_{0}\right]= \\
& 2 \exp \left(-j \omega 3 \pi / 2 \omega_{0}\right)
\end{aligned}
$$

in $-\sigma / 2<\omega<\sigma / 2$. It is easy to check that conditions $(17 \mathrm{a}, \mathrm{b})$ are satisfied. Then, applying the previous formulation, we arrive finally at

$$
\begin{aligned}
& b_{y_{1}}(t)=i_{y_{1}}(t)=\frac{\sin (\sigma t / 4+\pi / k)}{\sin (\pi / k)} \frac{\sin (\sigma t / 4)}{\sigma t / 4} \\
& b_{y_{2}}(t)=i_{y_{2}}(t)=\frac{\sin (\sigma t / 4)}{\sin (\pi / k)} \frac{\sin \left[\sigma\left(t+\pi / \omega_{0}\right) / 4\right]}{\sigma\left(t+\pi / \omega_{0}\right) / 4} \\
& b_{y_{3}}(t)=j q_{y_{3}}(t)=j \frac{\sin (\sigma t / 4+3 \pi / 2 k)}{\sin (\pi / k)}
\end{aligned}
$$

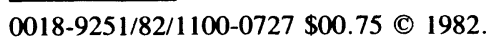




$$
\begin{gathered}
\cdot \frac{\sin \left[\sigma\left(t+\pi / 2 \omega_{0}\right) / 4\right]}{\sigma\left(t+\pi / 2 \omega_{0}\right) / 4} \\
b_{y_{4}}(t)=j q_{y_{4}}(t)=j \frac{\sin (\sigma t / 4+\pi / 2 k)}{\sin (\pi / k)} \\
\frac{\sin \left[\sigma\left(t+3 \pi / 2 \omega_{0}\right) / 4\right]}{\sigma\left(t+3 \pi / 2 \omega_{0}\right) / 4} .
\end{gathered}
$$

Inserting (25a-d) in (22), we obtain the corresponding interpolation formula.

Note that the considered linear systems are pure delays and that we can easily extend the method for $N>2$. Obviously this is a case of great interest that constitutes a strict generalization of Grace-Pitt's interpolation formula.

\section{WHEN GENERALIZED BROWN'S CONDITION IS NOT SATISFIED}

When $\omega_{0} \neq k \sigma / 2 N$ and $\omega_{0}$ must be maintained in (22) because it is a carrier frequency or there is some symmetry with respect to $\omega_{0}$, we can use the smallest $\sigma^{\prime}>\sigma$ such that $\omega_{0}=k \sigma^{\prime} / 2 N$. This value is given by

$\sigma^{\prime}=2 N \omega_{0} / E\left[2 N \omega_{0} / \sigma\right]$

Where $E[\cdot]$ indicates the greatest integer function. The corresponding new sampling rate is

$\sigma^{\prime} / 2 \pi N=1 / T^{\prime}=\omega_{0} / \pi E\left[2 N \omega_{0} / \sigma\right]$.

$\sigma^{\prime}$ and $T^{\prime}$ take the places of $\sigma$ and $T$, respectively, in (22).

Since band limitation is not a physical possibility, in a general situation $X$ does not have center angular frequency in a strict sense. Thus we can search for the minimum sampling rate without maintaining $\omega_{0}$. It is not difficult to prove that the corresponding new bandwidth $\sigma^{\prime \prime}$, sampling period $T^{\prime \prime}$, and center angular frequency $\omega_{0}^{\prime \prime}$ are given by [17]

$$
\begin{aligned}
\sigma^{\prime \prime}= & \left(2 N \omega_{0}+\sigma\right) / E\left[\left(2 N \omega_{0}+\sigma\right) / \sigma\right] \\
T^{\prime \prime}= & 2 \pi N / \sigma^{\prime \prime}=2 \pi N E\left[\left(2 N \omega_{0}+\sigma\right) / \sigma\right] /\left(2 N \omega_{0}\right. \\
& +\sigma)>T^{\prime}=2 \pi N / \sigma^{\prime} \\
= & 2 \pi N E\left[\left(2 N \omega_{0}+\sigma\right) / \sigma\right] /\left(2 N \omega_{0}+\sigma^{\prime}\right)
\end{aligned}
$$

and

$\omega_{0}^{\prime \prime}=\omega_{0}+\sigma / 2-\sigma^{\prime \prime} / 2$.

$\sigma^{\prime \prime}, T^{\prime \prime}$, and $\omega_{0}^{\prime \prime}$ take the places of $\sigma, T$, and $\omega_{0}$, respectively, in (22).

\section{EQUIVALENCE WITH MODIFIED PAPOULIS' FORMULATION}

It is possible to verify that the solutions $\left\{Y_{i}(\omega, t)\right\}, i$

$=1, \ldots, 2 N$, of (3) can be obtained as linear time-vary- ing combinations of the pieces of each previously obtained solution in a way parallel to that indicated by Brown in the low pass case. In particular this ensures the equality of the previous $\left\{y_{i}(t)\right\}$ with the $\left\{y_{i, 0}(t)\right\}$ of [17], $i=1, \ldots, 2 N$, and of the $\left\{i_{v_{i}}(t)\right\}, i=1, \ldots, N$, and the $\left\{q_{v_{i}}(t)\right\}, i=N+1, \ldots, 2 N$, of this paper with the $\left\{z_{i, 0}(t)\right\}$, $i=1, \ldots, N$, and the $\left\{-j z_{i, 0}(t)\right\}, i=N+1, \ldots, 2 N$, of [17], respectively, as it is necessary.

We omit the corresponding proof for brevity, since it is an immediate generalization of $[19$, sec. III $]$.

\section{CONCLUSIONS}

Starting from Brown's formulation of multichannel sampling of bandpass signals, we have introduced a general separate interpolation formula to reconstruct the signal from the samples of the outputs of $2 \mathrm{~N}$ linear, timeinvariant systems driven by it taken at a rate $1 / 2 \mathrm{~N}$ times the Nyquist rate, under the following conditions:

1) The transfer functions of the systems have to generate a nonsingular matrix $\boldsymbol{A}$ (12).

2) The center angular frequency and the bandwidth of the signal are related by $\omega_{0}=k \sigma / 2 N$, where $k$ is an integer (generalized Brown's condition).

3) The impulse responses of $N$ systems have zero quadrature components, while the impulse responses of the other $N$ systems have zero in-phase components.

The proposed formulation has the advantage with respect to others previously stated of offering the transfer functions of the interpolating filters directly.

An interesting example, in which all the linear systems are pure delays, is considered and solved to illustrate the proposed method.

When generalized Brown's condition is not satisfied, we can apply this method with a minimum sampling rate $1 / T^{\prime}=\sigma^{\prime} / 2 \pi N=\omega_{0} / E\left[2 N \omega_{0} / \sigma\right]$ maintaining the center angular frequency, or with a minimum sampling rate $1 / T^{\prime \prime}$ $=\sigma^{\prime \prime} / 2 \pi N=\left(2 N \omega_{0}+\sigma\right) / 2 \pi N E\left[\left(2 N \omega_{0}+\sigma\right) / \sigma\right]$ without maintaining $\omega_{0}$.

\section{ACKNOWLEDGMENTS}

The authors wish to thank Prof. A. Papoulis for introducing them to the generalized sampling expansions theory and Prof. J.L. Brown, Jr., for focusing their attention on his multichannel sampling works and for helpful discussions about this subject.

\author{
ANÍBAL R. FIGUEIRAS-VIDAL \\ JOSÉ R. CASAR-CORREDERA \\ ETSI Telecomunicacion-UPM \\ Ciudad Universitaria \\ Madrid 3, Spain
}

MIGUEL A. LAGUNAS-HERNÁNDEZ RAMÓN GARCÍA-GÓMEZ

ETSI Telecomunicacion-UPB Jorge Girona Salgado $\mathbf{s} / \mathbf{n}$ Barcelona 34, Spain 
[1] Brown, J.L., Jr. (1980) First-order sampling of bandpass signals-A new approach. IEEE Transactions on Information Theory, 1980, IT-26, 613-615.

[2] Whittaker, E.T. (1915)

On the functions which are represented by the expansion of interpolating theory.

Proceedings of the Roval Societv, Edinburg, 1915, 35, 181194

[3] Shannon, C.E. (1949)

Communication in the presence of noise

Proceedings of the IRE, 1949, 37, 10-21.

[4] Goldman, S. (1953)

Information Theory.

Englewood Cliffs, N.J.: Prentice-Hall, 1953.

[5] Kohlenberg, A.W. (1953)

Exact interpolation of band-limited functions.

Journal of Applied Phvsics, 1953, 24, 1432-1436.

[6] Linden, D.A. (1959)

A discussion of sampling theorems.

Proceedings of the IRE, 1959, 47, 1219-1226.

[7] Grace, O.D., and Pitt, S.P. (1968)

Quadrature sampling of high-frequency waveforms.

Journal of the Acoustical Society of America, 1968, 44, 1453-1454.

[8] Persons, C.E. (1975)

Quadrature sampling error formula.

Journal of the Acoustical Society of America, 1975, 57, 511512.

[9] Brown, J.L., Jr. (1979)

On quadrature sampling of bandpass signals.

IEEE Transactions on Aerospace and Electronic Systems. 1979, AES-15, 366-371.

[10] Brown, J.L., Jr. (1979)

Sampling bandpass waveforms for direct determination of lowpass components.

Proceedings of the 22nd Midwest Conference on Circuit Systems, 1979, 135-139.

[11] Brown, J.L., Jr. (1980)

A simplified approach to optimum quadrature sampling. Journal of the Acoustical Society of America, 1980, 67. 1659-1662.

[12] Figueiras-Vidal, A.R., Mariño-Acebal, J.B., Lagunas-Hernández, M.A., and García-Gómez, R. (1980)

Some new results in sampling deterministic signals.

Proceedings of the First European Conference on Signal Processing, 1980, 197-200.

[13] Figueiras-Vidal, A.R. (1981)

Sampling bandpass signals.

IEEE Transactions on Aerospace and Electronic Systems, Mar. 1981, AES-17, 288-295.

[14] Papoulis, A. (1968)

New results in sampling theory.

Presented at the Hawaii International Conference on Systems Science, 1968

[15] Papoulis, A. (1968)

Systems and Transforms with Applications in Optics.

New York: McGraw-Hill, 1968.

[16] Papoulis, A. (1977)

Generalized sampling expansion.

IEEE Transactions on Circuits and Systems, 1977, CAS-24, 652-654.

[17] Figueiras-Vidal, A.R., Mariño-Acebal, J.B., Lagunas-Hernández, M.A., and García-Gómez, R. (1981)

Reduction of individual sampling rates by generalizing quadrature methods of separate interpolation.

Journal of the Acoustical Society of America, 1981, 70, 1331-1335.
[18] Brown, J.L., Jr. (1979)

Generalized sampling of bandpass signals (abstract).

IEEE International Symposium on Information Theory, 1979, Grignano, Italy, 155

[19] Brown, J.L., Jr. (1981)

Multichannel sampling of lowpass signals.

IEEE Transactions on Circuits and Systems, 1981, CAS-28, 101-106

[20] Brown, J.L., Jr. (1982)

Sampling rate reduction in multichannel processing of bandpass signals

Journal of the Acoustical Society of America, 1982, 71, 378383.

[21] Franks, L.E. (1969)

Signal Theory.

Englewood Cliffs, N.J.: Prentice-Hall, 1969.

\section{MTI Processing and Weibull-Distributed Ground Clutter}

It is shown that the Weibull-distributed ground clutter obeys a Weibull distribution after processing by the double canceler moving target indicator (MTI).

In a previous paper by the authors [1], it was shown that ground clutter amplitude from cultivated land obeys a Weibull distribution with the shape parameters of 1.507 to 2.0 at very low grazing angles between $0.21^{\circ}$ and $0.32^{\circ}$ using an $L$ band long-range air-route surveillance radar with a $3.0 \mu \mathrm{s}$ pulsewidth and a $1.23^{\circ}$ beamwidth

The present paper investigates the Weibull statistics of ground clutter after double canceler MTI processing.

Four typical examples before and after MTI are shown in Fig. 1. A straight line was fitted to the values of $Y$ and $X$ by the least squares method. If the data follow

TABLE I

Shape Parameters for Different Azimuth Sectors (values in parentheses are those of rmse)

\begin{tabular}{cccc}
\hline \hline & & \multicolumn{2}{c}{ Shape Parameter } \\
\cline { 3 - 4 } Azimuth (deg) & Sumber & Before MTI & After MTI \\
\hline $90.57-92.56$ & $868-887$ & $2.011(0.114)$ & $1.758(0.080)$ \\
$92.66-94.64$ & $888-907$ & $1.843(0.116)$ & $1.731(0.050)$ \\
$94.75-96.73$ & $908-927$ & $1.833(0.055)$ & $1.783(0.058)$ \\
$96.83-98.82$ & $928-947$ & $1.858(0.069)$ & $1.734(0.025)$ \\
$98.92-100.90$ & $948-967$ & $1.941(0.125)$ & $1.728(0.096)$ \\
$101.01-102.99$ & $968-987$ & $1.820(0.102)$ & $1.859(0.050)$ \\
$103.10-105.08$ & $988-1007$ & $1.720(0.063)$ & $1.760(0.099)$ \\
$105.18-107.17$ & $1008-1027$ & $1.841(0.094)$ & $1.745(0.054)$ \\
$107.27-109.25$ & $1028-1047$ & $1.804(0.080)$ & $1.618(0.062)$ \\
$109.36-111.34$ & $1048-1067$ & $1.507(0.046)$ & $1.553(0.063)$ \\
$111.44-113.43$ & $1068-1087$ & $1.670(0.120)$ & $1.519(0.039)$ \\
\hline
\end{tabular}

Manuscript received August 4, 1981.

0018-9215/83/0100-0729\$00.75 C) 1983 IEEE 